\title{
INVESTIGATION OF THE VIOLAXANTHIN CYCLE
}

\author{
D. I. SAPOZHNIKOV
}

\author{
Botanical Institute of the Academy of Sciences, Leningrad
}

\begin{abstract}
This paper is dedicated to the problem of the role of the violaxanthin cycle in the transport of oxygen.

The first part of the article deals with the phenomenology of this cycle; the second part contains a review of biochemical experiments on the participation of violaxanthin in oxygen transfer, and in the third part a survey is made of the objections to the view that the violaxanthin cycle participates in oxygen evolution.

A hypothesis on the mechanism of the oxygen evolution in photosynthesis is described.
\end{abstract}

\section{INTRODUCTION}

The carotenoids represent the most widely distributed group of naturally occurring pigments: more than 300 different carotenoids are known in animals, plants and bacteria ${ }^{1,2}$. About a dozen carotenoids have been selected in the course of evolution of the plastids of green plants; three xanthophylls-violaxanthin, zeaxanthin and antheraxanthin-are involved in a series of photoinduced interconversions, which are known as the 'violaxanthin cycle ${ }^{3.4}$. This cycle consists of de-epoxidation of violaxanthin, the so-called light or direct reaction, and of epoxidation of zeaxanthin, which is also known as a back, or a dark reaction ${ }^{5,6}$; the violaxanthin cycle operates through an intermediate monoepoxyxanthophyll, antheraxanthin ${ }^{7,8}$.

The chemical structure of the xanthophylls involved in the violaxanthin cycle makes it probable that they participate in oxygen transport; the violaxanthin de-epoxidation could bring about the formation of molecular oxygen, thus being a part of the oxygen-liberating system in photosynthesis $^{9,10}$ (Figure 1). There have been a great many findings in favour of this concept, but there is still no generally accepted view about it ${ }^{11-13}$. In this paper I should like to discuss in detail the problem of the role of the violaxanthin reactions in the process of photosynthetic oxygen evolution ${ }^{14,15}$.

The first part of this article is dedicated to the phenomenology of the violaxanthin cycle. In the second part the biochemical experiments based on the use of isotopic-labelling techniques, 'physiological labels' and experiments with inhibitors, and other means of intervening in the normal course of the physiological process, such as cell disintegration, etc., will be considered. The last part of this paper consists of a critical survey of the 
objections to the possibility that violaxanthin participates in oxygen evolution in photosynthesis ${ }^{16-18}$.<smiles>CC(/C=C/C=C/C=C/C12OC1(C)C(C)(C)CC(O)CC2(C)C)=C\C=C\C=C(C)\C=C\C=C\C(C)=C\C=C\C12OC1(C)CC(O)CC2(C)C</smiles><smiles>CC1=C(/C=C/C(C)=C/C=C/C=C(C)/C=C/C=C/C=C(C)/C=C/C=C(C)/C=C/C2=C(C)CC(O)CC2(C)C)C(C)(C)CC(O)C1</smiles>

Figure I. The violaxanthin cycle

\section{PHENOMENOLOGY OF THE VIOLAXANTHIN CYCLE}

In this section we shall consider the violaxanthin-cycle reactions from a physiological point of view. To achieve this aim it has been necessary to study the action of three main factors-light, temperature and time- on the course of the de-epoxidation and epoxidation of xanthophylls ${ }^{19}$.

In a series of investigations by the Group for Pigment Study of the Botanical Institute in Leningrad it has been shown that the violaxanthin deepoxidation reaction depends on the light intensity ${ }^{20-22}$. A comparison of the photosynthetic light curves with those of the de-epoxidation of violaxanthin has disclosed a certain discrepancy between these two processes: while the photosynthesis begins at very low-light intensities, there exists a definite threshold of light intensity below which, under steady-state conditions, one cannot observe the formation of zeaxanthin ${ }^{11,19}$. The threshold level depends primarily on the relation of the plant to light; for instance, such shade-tolerant plants as hydrangea and palisota have a rather low level (c. $800-1000 \mathrm{~lx}$ ) whereas such light-tolerant plants as peas and corn have a rather high-threshold level $(c .8000-10000 \mathrm{~lx})$.

Study of the light curves of the violaxanthin-cycle reactions has disclosed the existence of three ranges: $(a)$ a region corresponding to light intensities below the threshold level; (b) a region corresponding to light intensities above the threshold, and (c) a region corresponding to light intensities saturating the de-epoxidation reaction (Figure 2).

The threshold level is strongly temperature dependent. A special study has shown that lowering the temperature shifts the threshold level in the direction of low intensities, and, vice versa, that increasing the temperature shifts the threshold level in the direction of higher intensities. For instance, at $20^{\circ} \mathrm{C}$ the threshold level for pea plants is $7000 \mathrm{~lx}$; lowering the temperature 


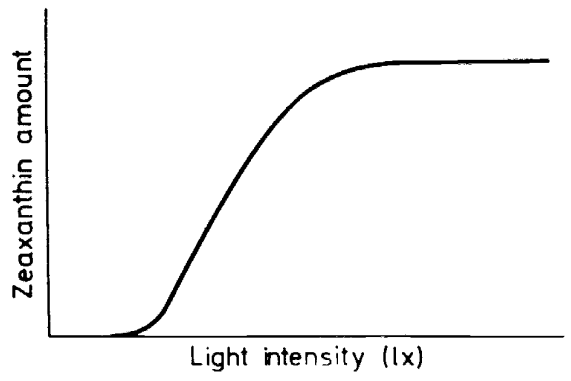

Figure 2. An idealized de-epoxidation light curve

to $10^{\circ} \mathrm{C}$ produces a shift of the threshold level to $4000 \mathrm{~lx}$, and a further decrease to $4^{\circ} \mathrm{C}$ shifts the level to $1000 \mathrm{~lx}$. Conversely, an increase in the temperature to $32^{\circ} \mathrm{C}$ results in a shift of the level to $8000 \mathrm{~lx}$.

In order to explain the phenomenon of the light threshold, as well as its temperature dependence, a hypothesis has been put forward according to which the phenomenon is a result of a compensation of the velocities of the direct and back reactions of the violaxanthin cycle. In order to check the correctness of this supposition, we inhibited the back reaction by means of salicylaldoxime ${ }^{23}$. The action of salicylaldoxime resulted in abolishing the light threshold of the violaxanthin de-epoxidation. Thus it has been shown that the violaxanthin cycle operates at light intensities even below the threshold level ${ }^{24}$.

At light intensities above the threshold level the velocity of the de-epoxidation reaction exceeds the velocity of the epoxidation thus making it possible to detect a certain amount of zeaxanthin. The quantity of zeaxanthin which is detected at still higher intensities is proportional to the intensity of light up to saturation point. The quantity of zeaxanthin produced at saturation intensities of light is nearly equal to half of the initial amount of violaxanthin ${ }^{5,7,11}$. This could be explained by assuming that at saturation intensity the amount of active violaxanthin is exhausted ${ }^{19}$.

The study of the action spectra of the violaxanthin-cycle reactions is of great importance for the physiology of this process.

As has been shown in a series of articles by several authors, the action spectrum of the violaxanthin de-epoxidation reaction coincides with the spectrum of chlorophyll absorption $7,25,26$. Especially important is the circumstance that the reactions of the violaxanthin cycle can be achieved in the red part of the visible spectrum.

The first experiments were carried out with broad red light; they showed that the de-epoxidation reaction of violaxanthin could be brought about if the intensity of the red light was sufficiently high ${ }^{27}$. A more detailed study of the action of different parts of the red light has revealed a different effect on the reactions of the violaxanthin cycle of near-red $(670 \mathrm{~nm})$ and far-red (704 nm) light ${ }^{28}$.

It has been shown that epoxidation of zeaxanthin, although being a dark reaction, can be stimulated by light; the intensity of light must be below the 


\section{I. SAPOZHNIKOV}

threshold level ${ }^{29}$. This phenomenon is especially well demonstrated in cases where sub-threshold intensities were used after illumination with saturating light intensities ${ }^{5}$.

To sum up the results obtained in experiments on the action of light, we may state that light intensities providing two thirds of the photosynthesis are still below the light threshold of the violaxanthin-cycle reactions ${ }^{11}$. In special experiments it has been shown that even sub-threshold intensities of light are sufficient for the reactions of the violaxanthin cycle, although it would be impossible to observe a change in the content of zeaxanthin ${ }^{11}$. It is advisable to take these facts into consideration when comparing the violaxanthin-cycle reactions with the different photosynthetic reactionsHill reaction, photophosphorylation and carbon dioxide reduction.

The time curves were investigated simultaneously and independently in the laboratories at Leningrad and Tubingen ${ }^{11,19}$. Our experiments at Leningrad were carried out on hydrangea, a shade-tolerant plant, while Lemna, a light-tolerant plant, was used in the other experiments.

It appeared that the shape of the light curves is dependent on the light intensity used in the experiment. Accordingly, there are three types of time curves depending on the light intensities under investigation.

The time course for saturating intensities is shown in Figure 3. At saturating light intensities the time curve increases rapidly up to the fifth minute when it reaches its maximum; prolonged exposures do not change the level attained at the fifth minute ${ }^{20}$.

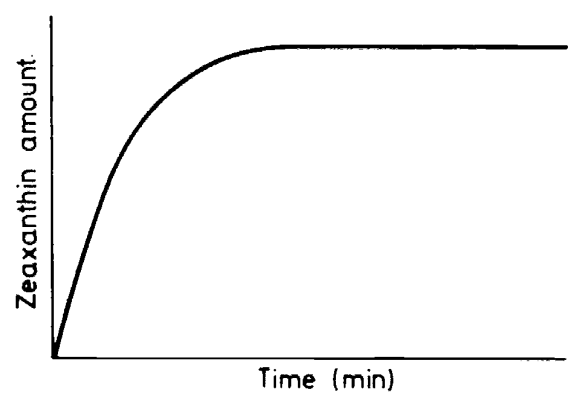

Figure 3 . The time curve at saturating light intensities

The time curve for intensities above the threshold (Figure 4) has the same maximum at the fifth minute; longer exposures produce a decrease in the content of zeaxanthin. The steady-state level is reached at the thirtieth minute; the level attained is dependent on the light intensity - the higher the intensity the nearer is the level to the saturation point ${ }^{11}$.

The time curve for intensities below the light threshold (Figure 5) has a small but distinct maximum at the fifth minute, the weaker the light intensity the lower the maximum level. At the thirtieth minute the initial dark level is attained ${ }^{17}$.

All these regularities are valid only when the light exposure lasts for at least 60 seconds. Shorter exposures disclose an induction period lasting from 2 to 5 seconds to 30 to 60 seconds. During the induction period one observes 


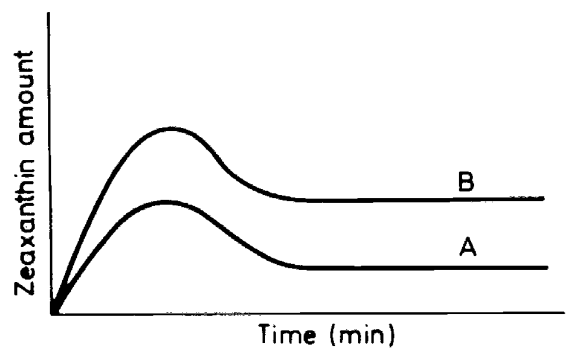

Figure 4. The time curves at intensities above the light threshold

a so-called 'unbalanced condition'19. This phenomenon reflects a certain incongruity between the specific components of the violaxanthin cycle. The induction period is the more distinct the lower the light intensity. The phenomenon of the induction period is far from being clearly understood; there is no one unambiguous explanation. One plausible hypothesis considers the above mentioned facts as 'tails' of the processes of super-rapid interconversions of carotenoids observed by different authors ${ }^{30-32}$.

Summing up all the facts known about the time dependence of the violaxathin-cycle reactions, we can say that it is a very complex function, its complexity being based on the fact that what we observe in our experiments may be a result of several different reactions with different velocity constants.

The investigation of the influence of temperature on the violaxathincycle reactions has revealed the following regularities.

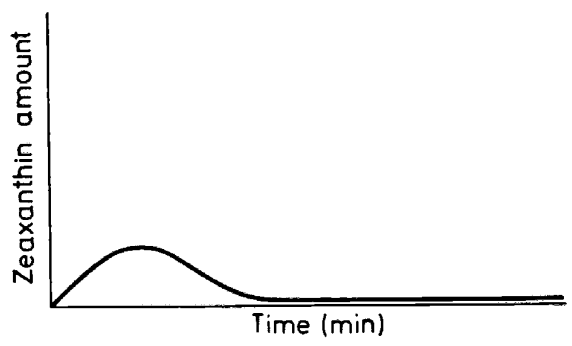

Figure 5. The time curve at sub-threshold light intensities

First, it has been shown that the epoxidation observed in the absence of light is strongly temperature dependent; thus, for instance, it is fully inhibited at $+2^{\circ} \mathrm{C}$. However, if the epoxidation reaction is observed under illumination, as a part of the violaxanthin cycle, it becomes less susceptible to temperature. This fact led to the supposition of the existence of two types of dark reactions. This hypothesis was confirmed later by using the specific inhibitors, salicylaldoxim and $p$-chlormercurybenzoate.

Second, as stated earlier, at constant temperature the threshold level is dependent on the light intensity; conversely, it has now been shown that, 
at constant light intensity, the threshold level is temperature dependent. Thus, for instance, the de-epoxidation reaction in pea leaves has been investigated at $6000 \mathrm{~lx}$. The results of such an experiment are depicted in Figure 6.

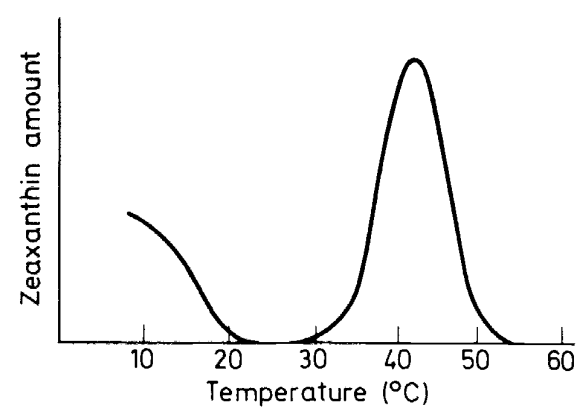

Figure 6. The temperature dependence of the de-epoxidation light curves

As can be seen from Figure 6, the temperature dependence curve consists of three regions. The middle part of the curve comprises the region adjacent to room temperature for which the light intensity used in this experiment is near the threshold; at such temperatures no zeaxanthin is observed. A decrease of temperature down to $+4^{\circ} \mathrm{C}$ discloses a shift in the compensation point as judged by the appearance of a considerable amount of zeaxanthin; at room temperature $6000 \mathrm{~lx}$ produce no zeaxanthin. At supra-optimal temperatures zeaxanthin appears as a result of the inactivation of the epoxidation enzyme; the maximum inactivation is attained at $42^{\circ} \mathrm{C}$. A further increase of temperature also leads to inactivation of the de-epoxidation; at $50^{\circ} \mathrm{C}$ a complete retardation is observable. It is noteworthy that no bleaching of violaxanthin occurs under these conditions-its quantity is the same both in the light and in the dark.

Thus the interaction of temperature and light intensity made it possible to study the course of both the de-epoxidation and epoxidation reactions under illumination.

The third peculiarity of the effect of temperature on the interconversion of xanthophylls is the role of the ecological properties of the plants studied. In the leaves of a tropical plant (Musa manii, $L$ ) the optimal temperature for the de-epoxidation reaction is shifted to a higher point $\left(40^{\circ} \mathrm{C}\right)$ when compared with the leaves of a plant of subtropical origin (Pelargonium zonale).

Summing up the results obtained through the study of the phenomenology of the violaxanthin cycle we are able to formulate three principal theses:

(1) There exists under steady-state conditions of the violaxanthin cycle a light threshold, i.e. a compensation point, where an equilibrium between the velocities of the dark and light reactions is maintained.

(2) In order to achieve the steady-state condition, in violaxanthin transformations, an induction period is necessary, the latter depending on the light intensity. 
(3) The enzymes participating in the de-epoxidation and epoxidation reactions of the violaxanthin cycle have a different susceptibility to temperature.

All these findings were disclosed by means of physiological investigations based on the analysis of the changes in the pigment concentrations. The knowledge of these facts is helpful in designing biochemical experiments with both indicators and inhibitors. The aim of these experiments is to obtain as much information as possible on the mechanism of violaxanthincycle reactions.

\section{BIOCHEMICAL INVESTIGATIONS OF THE VIOLAXANTHIN CYCLE}

The purpose of the biochemical investigations is to find out the mechanism of the reactions involved in the violaxanthin cycle, in particular the problem of participation of violaxanthin in oxygen transfer from the hydroxyl ion of the water molecule to molecular oxygen, i.e. the role of the violaxanthin cycle in oxygen evolution during the photosynthesis process. This problem consists of two parts. In the first place it was important to find out whether the hydroxyl ion of the water molecule participates as an oxygen donor for zeaxanthin. On the other hand, it was necessary to investigate whether violaxanthin could serve as a donor of molecular oxygen. Both of these questions could be answered by means of labelling experiments, particularly with an isotopic label.

The first experiments with heavy-oxygen water were carried out in $1951^{14}$. These experiments showed that in the presence of water labelled with heavy oxygen a suspension of Chlorella includes ${ }^{18} \mathrm{O}$ in the xanthophyll fraction when illuminated. Later experiments on Chlorella and Elodea showed that the water oxygen labels the violaxanthin molecule ${ }^{33}$. In order to ascertain that the heavy oxygen of the water labels the epoxide groups in the violaxanthin molecule a comparison has been made with the degree of labelling present in the fraction containing the dihydroxycarotenes; these experiments have actually shown that the hydroxy groups are not labelled with heavy oxygen of the water ${ }^{34}$. As Hager justly remarked another explanation of the results is possible. He thinks that the oxygen label in the violaxanthin molecule originates not directly from water but from the photosynthetically produced molecular oxygen ${ }^{18}$. If this were the case the violaxanthin-cycle reactions ought to be realized as follows from the reactions:

$$
\begin{gathered}
\text { Zeaxanthin }+{ }^{18} \mathrm{O}_{2} \stackrel{\text { dark }}{\rightarrow} \rightarrow{ }^{18} \mathrm{O} \text {-Violaxanthin } \\
{ }^{18} \mathrm{O} \text {-Violaxanthin }+4 \mathrm{H} \stackrel{\text { light }}{\rightarrow} \text { Zeaxanthin }+2 \mathrm{H}_{2}{ }^{18} \mathrm{O}
\end{gathered}
$$

As can be seen from the equations the labelling ought to be higher in the dark than in the light; in our experiments the reverse was the case, and therefore the process ought to proceed as follows:

$$
\begin{gathered}
\text { Zeaxanthin }+2 \mathrm{H}_{2}{ }^{18} \mathrm{O} \stackrel{\text { dark }}{\longrightarrow}{ }^{18} \mathrm{O} \text {-Violaxanthin }+4 \mathrm{H} \\
{ }^{18} \mathrm{O} \text {-Violaxanthin } \stackrel{\text { light }}{\longrightarrow} \text { Zeaxanthin }+{ }^{18} \mathrm{O}_{2}
\end{gathered}
$$




\section{I. SAPOZHNIKOV}

Thus, the experiments carried out with water labelled with heavy oxygen led us to the conclusion that first, the oxygen in the epoxide groups of the violaxanthin molecule originates at least partially from the hydroxyl ion of water, and secondly that on illumination there is an increase of the labelled oxygen in the violaxanthin molecule ${ }^{34}$.

Thus we could assert that the photosynthetic oxygen which has its origin in water molecules might be transferred through the carotenoids. These experiments, however, do not allow us to assume that all of the oxygen liberated in photosynthesis migrates through the violaxanthin cycle.

A closer approach to the solution of this problem would be a comparison of the labelling of the water medium, the intracellular water, the violaxanthin and the molecular photosynthetic oxygen. As follows from specially performed experiments, the labelling of the violaxanthin oxygen is only one third of that of the molecular oxygen ${ }^{35}$. These results could be easily explained by assuming that, firstly, not all the molecules of violaxanthin are active in the de-epoxidation reactions, and, secondly, by taking into consideration the heterogeneity of violaxanthin ${ }^{36}$. If we could relate the labelling to only some of the violaxanthin molecules, the extent of labelling would equal that of the molecular oxygen.

To obtain a deeper insight into the problem of violaxanthin participation in oxygen transport in photosynthesis a kinetic experiment has been carried out $^{38}$. This experiment was designed in such a way as to follow the time course of labelling the epoxide groups of violaxanthin.

These experiments have shown that, in the presence of heavy-oxygen water (73 per cent enrichment), a suspension of Chlorella cells introduces the labelled oxygen into the epoxide groups of violaxanthin within 30 minutes in the dark. This result shows that the dark reaction-epoxidation-is accompanied by inclusion of water oxygen in the violaxanthin molecule. By the same token we may repudiate Hager's remark about the photosynthetic origin of the epoxide groups in violaxanthin.

Subsequent illumination of the suspension for two seconds causes a drastic decrease of the oxygen labelling of the violaxanthin molecule. By extrapolation we concluded that the de-epoxidation reaction begins after two hundredths of a second.

Further increase in the illumination time up to 60 seconds leads to an increase in the labelling followed by a subsequent decrease during five minutes. Beginning from the fifth minute a steady increase in the labelling occurred reaching the initial value at the twelfth minute; after 30 minutes of illumination the labelling was double that observed initially.

This experiment confirms the findings obtained in the physiological experiments which demonstrated the existence of an induction period in the interconversion of the xanthophylls constituting the violaxanthin cycle. Besides, it also confirms the phenomenon observed earlier that the labelling increases in illuminated leaves as compared with the darkened ones. Consequently the kinetic experiment supports the view that the reactions of the violaxanthin cycle occur by way of the photo-oxidation of the epoxide group. Furthermore, it is apparent that the kinetic experiment demonstrates the steady-state formation of the violaxanthin cycle by gradually increasing the amounts of violaxanthin which became active in the reactions. 
Subsequent biochemical investigations were carried out in order to elucidate the place of the violaxanthin cycle in the process of photosynthesis. Special experiments have shown the heterogeneity of violaxanthin in the plastids of green plants. In order to demonstrate this fact we used differential centrifugation to give two fractions corresponding to the two photosystems. In addition, we have marked the violaxanthin in different fractions with the so-called 'physiological label' ${ }^{36}$. This method consists of illumination of the leaves before they are homogenized; in such leaves one can observe simultaneously both violaxanthin and zeaxanthin. As an index of heterogeneity of the photochemical activity one can use the relative amounts of these pigments in separate fractions. It has appeared that in strong light $(80000 \mathrm{~lx})$, in the light fraction corresponding to the photosystem I, the physiological label was from two to three times higher than that in the heavy fraction which corresponds to the photosystem II. This fact may be explained by assuming that at high-light intensities the epoxidation reaction in the photosystem I is retarded as a result of a local anaerobiosis. That this is the case has been shown by decreasing the light intensity; the decrease of light intensity led to equalization of the physiological label in both fractions.

The existence of two different pools of violaxanthin in the chloroplasts corresponds to the presence of two alternative ways of violaxanthin deepoxidation.

The first indications of the existence of two ways of de-epoxidation were obtained in experiments in which the interaction of two factors was studied, the factors being anaerobiosis and hydroxylamine. The latter is an inhibitor of the oxygen-liberating link of the photosynthesis.

It appeared that while hydroxylamine inhibits the de-epoxidation under aerobic conditions, no inhibitory action is observed under anaerobiosis ${ }^{37}$. These findings have allowed us to confirm the suggestion made by Calvin in 1959 that there may be two different ways of de-epoxidation ${ }^{4}$; the photooxidation of the epoxy groups in violaxanthin leads to the evolution of molecular oxygen, and the photo-reduction of the same groups leads to the formation of water molecules. Subsequently, analogous experiments were carried out with Diuron-another inhibitor of the oxygen-liberating link of the photosynthesis ${ }^{39}$. Low concentrations of this inhibitor $\left(10^{-7} \mathrm{M}\right)$ induce a retardation of the de-epoxidation up to 50 per cent, whereas high concentrations $\left(10^{-5} \mathrm{M}\right)$ inhibit 100 per cent of this reaction. Like hydroxylamine, Diuron does not inhibit the de-epoxidation reaction under anaerobiosis. Thus, the experiments carried out with Diuron have shown that low concentrations of this inhibitor, which are affecting the reaction of the photosystem II-non-cyclic photophosphorylation and oxygen evolutionalso inhibit about half of the de-epoxidation reaction. High concentrations of Diuron, which affect the reactions of the first photosystem-cyclic photophosphorylation-completely inhibit the de-epoxidation.

Experiments on greening seedlings of Zea mais have also shown that there are two ways of de-epoxidation ${ }^{40}$. It is well known that etiolated seedlings contain violaxanthin and other carotenoids as well as protochlorophyll ${ }^{41}$. In order to perform the de-epoxidation, however, the accumulation of a certain amount of chlorophyll is necessary; 1-2 hours of greening is required to initiate de-epoxidation reactions ${ }^{42,43}$. For the completion of the whole 


\section{I. SAPOZHNIKOV}

violaxanthin cycle it is necessary to have a longer greening time of up to 5-6 hours, at which time the formation of the photosynthetic apparatus is accomplished. Low concentrations of Diuron completely inhibit the deepoxidation reaction in seedlings greening for 1-2 hours ${ }^{40}$. After 5-6 hours of greening the same concentrations of Diuron inhibit only a part of this reaction.

The realization of the violaxanthin cycle under steady-state conditions is accomplished only when both the de-epoxidation and the epoxidation reactions are fulfilled. Up to now we could judge the fulfilment of the epoxidation reaction only after illumination of the objects under investigation in strong light, its intensity being either saturating or above the threshold; under these conditions a certain amount of zeaxanthin is accumulated, and after switching the light off it was possible to follow the decrease in the amount of this pigment as a result of the epoxidation reaction. Several authors are of the opinion that at sub-threshold intensities no interconversion of xanthophylls takes place ${ }^{11}$. Experiments with salicylaldoxime have shown that the violaxanthin cycle is also fulfilled at sub-threshold light intensities ${ }^{23}$. At these light intensities the rates of both the direct and dark reactions are balanced, resulting in a compensation point. An increase in light intensity results in an acceleration of the light reaction to such an extent that the back reaction is left behind; therefore there is an accumulation of zeaxanthin, which is easy to observe on a thin-layer chromatogram.

Since the epoxidation reaction is dependent on aerobic conditions it was important to investigate the role of oxygen concentration in the gaseous phase. Special experiments have been carried out, which have shown that an increase in oxygen concentration stimulates the epoxidation reaction resulting in a shift of the pigment content towards the more oxygenated violaxanthin.

The epoxidation reaction is the more sensitive of the two reactions to factors which tend to impair the violaxanthin-cycle integrity ${ }^{45}$. The deepoxidation reaction is less sensitive to different impairing agents; this reaction could be observed in isolated chloroplasts ${ }^{45}$, under anaerobiosis ${ }^{21}$, in artificial lipoproteid complexes ${ }^{46,47}$, and in pigment solutions $s^{48}$. Hager succeeded in isolating and partially purifying an enzyme-de-epoxidase. This enzyme catalyses the de-epoxidation reaction in vitro ${ }^{48}$.

Summing up the findings obtained in the course of the biochemical investigations and taking into consideration the results of the physiological studies, we come to the conclusion that in green plastids the violaxanthin cycle takes part in oxygen transport. The oxygen-transport process is of a dual nature, the epoxy atoms having an oxidation level intermediate between the hydroxyl ion of water and molecular oxygen. The photo-reduction of the epoxy atom leads to the formation of a water molecule, whereas the photo-oxidation of this atom results in oxygen evolution.

It has been shown earlier that the formation of the epoxy group is dependent on the presence of water, as well as on the presence of molecular oxygen. The participation of molecular oxygen in the formation of an epoxy group can be compared with the necessity of molecular oxygen for the beginning of the process of photosynthesis.

It is well known that under strictly anaerobic conditions a steadfast 


\section{INVESTIGATION OF THE VIOLAXANTHIN CYCLE}

inhibition of photosynthesis is observed ${ }^{49}$. The dark incubation of certain photosynthetic organisms in a hydrogen atmosphere causes an adaptation to this gas: under weak-light illumination, in the presence of carbon dioxide, such adapted-to-hydrogen organisms are able to assimilate this substance by way of photo-reduction, in the same way as purple sulphur bacteria ${ }^{50}$.

The transition from photo-reduction to photosynthesis might be accomplished in two ways: either by the addition of molecular oxygen, or by an increase of the light intensity. It should be noted, that the photochemical de-adaptation is inhibited by hydroxylamine; it has been shown earlier that hydroxylamine inhibits the photo-oxidation of the epoxy group of violaxanthin $^{51}$.

A correct interpretation of the phenomenon of photochemical deadaptation is of great importance for the understanding of the processes of photosynthesis; one can suggest that violaxanthin is the intermediate which, by photo-oxidation leads to oxygen evolution. With this suggestion in mind one can explain the process of photochemical de-adaptation as follows ${ }^{52}$.

At small light intensities under anaerobiosis the epoxy group of violaxanthin is photo-reduced, by the de-epoxidase enzyme, to water; this process presumably takes place in the photosystem I. An increase of the light intensity leads to the activation of a greater number of violaxanthin molecules; some of these molecules undergo photo-oxidation and produce a catalytic quantity of oxygen molecules, the latter being reproduced autocatalytically in a kind of a chain reaction.

This supposition about the course of photochemical de-adaptation is in good agreement with the inhibiting effect of hydroxylamine on this deadaptation; hydroxylamine inhibits the light reaction of the violaxanthin cycle, which is being accomplished by photo-oxidation. As a result of this inhibition it becomes impossible to produce even catalytic amounts of oxygen.

In order to explain the possibility of the autocatalytic increase of the molecular oxygen concentration we propose the following hypothesis. The hypothesis is based on the supposition that the molecular oxygen itself serves as an oxidant of water in the photosynthetic process. According to this hypothesis we assume that, for each molecule of absorbed molecular oxygen, two molecules of molecular oxygen are produced.

Schematically this process might be expressed as follows:

(a) the epoxidation reaction proceeds according to the equation

$$
2 \text { Zeaxanthin }+\mathrm{O}_{2}+2 \mathrm{H}_{2} \mathrm{O} \stackrel{\text { dark }}{\longrightarrow} 2 \text { Violaxanthin }+4 \mathrm{H}
$$

(b) the de-epoxidation reaction takes place according to the equation

$$
2 \text { Violaxanthin } \stackrel{\text { light }}{\longrightarrow} 2 \text { Zeaxanthin }+2 \mathrm{O}_{2}
$$

To sum up, we can see from these two equations that one molecule of oxygen plus two molecules of water produce four atoms of hydrogen and two molecules of oxygen.

The participation of violaxanthin in oxygen transfer during photosyn- 
thesis does not exclude its possible participation in other reactions of photosynthesis such as, for instance, photosynthetic photophosphorylation ${ }^{18,53 \cdots 55}$.

\section{A CRITICAL SURVEY OF THE OBJECTIONS TO THE POSSIBLE PARTICIPATION OF THE VIOLAXANTHIN CYCLE IN OXYGEN EVOLUTION IN PHOTOSYNTHESIS}

It is now a universally accepted fact that the violaxanthin cycle takes part in the process of photosynthesis; however, there is no agreement about the position of this cycle in the chain of events ${ }^{56}$. This section is an exposition of our critique of the objections to the hypothesis that the violaxanthin cycle participates in the oxygen evolution. These objections are of a dual nature; part of them are based on theoretical considerations, and part on the experimental findings. The theoretical objections are concerned with what seems to be a conflict with the laws of chemistry and physics. Below we shall discuss these contradictions separately.

The carotenoids absorb in the blue region of the visible spectrum and do not absorb in the red region ${ }^{57}$. Earlier it was shown that the violaxanthincycle reactions could proceed in red light. The necessity of chlorophyll for the operation of the violaxanthin cycle has been demonstrated in experiments on greening seedlings ${ }^{58}$. These circumstances give rise to a theoretical contradiction; on the one hand the carotenoids do not absorb in the red region of visible light, and on the other hand the oxygen evolution can proceed in red light. It is well known that the excitation energy can be transferred only from pigments absorbing at shorter wavelengths to pigments which absorb at longer wavelengths.

In order to explain the possibility of a transfer of excitation energy from the green pigments to the yellow ones, several hypotheses have been proposed ${ }^{59}$. The most probable one is based on the supposition that the energy transfer from the chlorophylls to carotenoids becomes possible on the triplet level of excitation of these pigments ${ }^{60}$. During the last two years the energy transfer from chlorophylls to the carotenoids has been demonstrated experimentally $30,31,60$. These experiments were carried out using laserflash techniques.

Concluding this part of the critical survey we might say that at present there are no contradictions with the laws of physics in the hypothesis that the violaxanthin cycle participates in oxygen evolution.

Now we shall discuss the objections to the participation of the violaxanthin cycle in oxygen evolution from the chemical point of view.

The authors who regard it as impossible that oxygen evolution is achieved by means of the violaxanthin cycle base their objections on the grounds that the de-epoxidation is accomplished only as a photo-reduction of the epoxy group of the violaxanthin molecule; they do not regard the photo-oxidation of the epoxy group in the violaxanthin molecule as being possible.

In order to be able to consider with assurance the possibility of the photooxidation of the epoxy groups of violaxanthin it was necessary to find an analogous chemical model. Ethylene oxide, the simplest epoxide, serves as such a model. The latest experiments on this epoxide have shown that ultraviolet radiation is able to disrupt both the carbon-carbon and carbon- 


\section{INVESTIGATION OF THE VIOLAXANTHIN CYCLE}

oxygen bonds. By using flash techniques it was possible to show that about a third of the photoproducts can be trapped as two-carbon compounds ${ }^{61}$. These experiments have shown unambiguously the possibility of photooxidation of the epoxy atom of this simple epoxide.

Our line of reasoning is based on these experiments; we regard it as possible that the violaxanthin molecule can be photo-oxidized when illuminated with visible light, in the same way as ethylene oxide.

Thus, we conclude this part of the critical review by stating that it is quite possible for the violaxanthin cycle to participate in the oxygen evolution in photosynthesis; the objections made from the physical point of view are being nullified by the experiments on excitation energy transfer in the triplet state, and the objections which were made on the grounds of chemical considerations are repudiated by the model experiments with ultraviolet radiation.

Now let us turn to the objections which are based on experimental findings.

The attention of the reader should be directed to the circumstance that the overwhelming majority of these findings were obtained from experiments at a time when the phenomenology of the violaxanthin cycle was not known. Thus the literature contained no reference to compensation point of direct and back reactions, sub-threshold light intensity, heterogeneity of violaxanthin, or induction period.

In a series of experiments a comparison was made of the de-epoxidation of violaxanthin with the photosynthesis process, the latter having been measured either by the oxygen evolution ${ }^{62}$ or by the carbon dioxide assimilation ${ }^{19}$. In both cases there was a discrepancy between the rate of de-epoxidation and the photosynthesis. These findings are now easily explained in the light of our knowledge about the xanthophyll interconversions which take place at light intensities below the compensation point.

Experiments with heavy-oxygen water were frequently carried out without taking into account either the induction period or the compensation point. Let us consider for instance the experiment of Shneour and Calvin ${ }^{63}$. They noted higher labelling in the dark sample as compared with the illuminated one. They designed their experiment without knowledge of the phenomenology of the violaxanthin cycle. Thus, the dark sample was incubated with heavy-oxygen water for 30 minutes, whereas the light sample was illuminated for 5 minutes, after 25 minutes of dark incubation. Now it is well known that the de-epoxidation of violaxanthin is reaching its maximum at the fifth minute; this means that the experimental conditions of this experiment ought to lead to the lowest labelling in the illuminated sample.

In analogous experiments, and in experiments which were carried out to compare the labelling in violaxanthin with the labelling in the molecular oxygen, the heterogeneity of violaxanthin has not been taken into consideration $^{35}$.

It has been shown in several papers that there exists a correlation between the de-epoxidation of violaxanthin and the photophosphorylation ${ }^{18,53-55}$. These findings can be explained on the basis of two pathways for violaxanthin de-epoxidation. It is probable that in those experiments a photo-reduction of the epoxy groups was recorded the more that high-light intensities were used. 


\section{I. SAPOZHNIKOV}

\section{CONCLUDING REMARKS}

The existence of the violaxanthin cycle was discovered 17 years ago. Since then much has been done to elucidate this phenomenon, and to study the mechanism of its reactions. There has been a steady increase in the number of laboratories which are studying the violaxanthin cycle.

There are three different hypotheses on the role of the violaxanthin cycle. According to the first hypothesis its role consists of the protection of the photosynthetic apparatus from strong light ${ }^{12}$. The second hypothesis postulates the correlation of the violaxanthin cycle reactions with photophosphorylation ${ }^{18}$. We are especially interested in the third hypothesis according to which the violaxanthin cycle plays an important role in oxygen evolution.

The present level of our knowledge does not allow us to exclude any of these possibilities. Let us hope that further investigations will be helpful in resolving this problem.

\section{REFERENCES}

1 T. W. Goodwin, Ann. Rev. Plant Physiol., 12, 219 (1961).

${ }^{2}$ O. Isler, Carotenoids, Birkhäuser Verlag, Basel (1971).

${ }^{3}$ H. J. Yamamoto, C. O. Chichester and T. O. M. Nakayama, Arch. Biochem. Biophys., 96, 645 (1962).

4 U. Blass, J. M. Anderson and M. Calvin, Plant Physiol., 34, 329 (1959).

5 A. Hager, Planta, 76, 138 (1967).

6 H. J. Yamamoto, C. O. Chichester and T. O. M. Nakayama, Photochem. Photobiol., 1, 53 (1962).

7 A. Hager, Planta, 74, 148 (1967).

${ }^{8}$ N. I. Krinsky, Biochim. Biophys. Acta, 88, 487 (1964).

D. I. Sapozhnikov, Studia Biophys., 5, 203 (1967).

10 D. I. Sapozhnikov, Progress in Photosynthesis Research, p. 694, (ed. H. Metzner), Tübingen (1969).

11 T. Siefermann, Über den Zusammenhang von Xanthophyllcyclus und Photosynthese bei Lemna gibba L., Thesis, Tübingen (1969).

12 N. I. Krinsky, Carotenoids, p. 669, (ed. O. Isler), Basel (1971).

13 A. Hager, Ber. Deut. Botan. Ges., 79, 94 (1966).

14 C. D. Dorough and M. Calvin, J. Am. Chem. Soc., 73, 2362 (1951).

15 D. I. Sapozhnikov, C. A. Dilova and L. V. Ivantsova, Dokl. Akad. Nauk USSR, 180, 742 (1968).

16 H. J. Yamamoto, T. O. M. Nakayma and C. O. Chichester, Arch. Biochem. Biophys., 97, 168 (1962).

17 H. J. Yamamoto, J. Chang and M. Aihara, Biochem. Biophys, Acta, 141, 342 (1967).

18 A. Hager, Planta, 89, 224 (1969).

19 I. A. Popova, F. F. Rhyzhova and D. I. Sapozhnikov, Dokl. Akad. Nauk USSR, 201, 494 (1971).

20 D. I. Sapozhnikov, T. A. Krasovskaya and A. N. Mayevskaya, Dokl. Akad. Nauk USSR, 113, 465 (1957).

21 D. I. Sapozhnikov, A. N. Mayevskaya, T. A. Krasovskaya-Antropova, L. A. Prialgauskajte and V. S. Turchina, Biokhimiya, 24, 39 (1959).

22 D. I. Sapozhnikov, Z. M. Eidelman, N. V. Bazhanova, T. G. Maslova and O. F. Popova, Eksperim. Botan., 15, 43 (1962).

23 D. I. Sapozhnikov, I. A. Popova and F. F. Rhyzhova, preprint, Leningrad (1971).

24 D. I. Sapozhnikov, I. A. Popova and F. F. Rhyzhova, Abstracts of the Fourth International Biophysical Congress, Moscow (1972).

${ }^{25}$ K. H. Lee and H. J. Yamamoto, Photochem. Photobiol., 7, 101 (1968).

26 D. I. Sapozhnikov, L. R. Kolotova and Yu. R. Giller, Dokl. Akad. Nauk USSR, 171, 740 (1966). 


\section{INVESTIGATION OF THE VIOLAXANTHIN CYCLE}

27 D. I. Sapozhnikov, Biochemistry and Biophysics of Photosynthesis, p. 190, Moscow (1965).

28 D. I. Sapozhnikov, I. A. Popova, T. G. Maslova and O. Ya. Korolyova, Dokl. Akad. Nauk USSR, 198, 1465 (1971).

29 C. Costes, Ann, Physiol. Vég., 10, 171 (1968).

${ }^{30}$ P. Mathis, C.R. Akad. Sci., Ser. D, 263, 1770 (1966).

${ }^{31}$ P. Mathis and J. M. Galmiche, C.R. Acad. Sci., Ser, D, 264, 1903 (1967).

${ }^{32}$ C. Wolff and H. T. Witt, Z. Naturforsch., 24b. 1031 (1969).

33 D. I. Sapozhnikov, D. G. Alkhazov, Z. M. Eidelman, N. V. Bazhanova, J. K. Lemberg, T. G. Maslova, A. B. Girshin, I. A. Popova, V. S. Saakov, O. F. Popova and G. A. Shirjayeva. Botan. Zh., 46, 673 (1961).

34 D. I. Sapozhnikov, D. G. Alkhazov, Z. M. Eidelman, N. V. Bazhanova, J. K. Lemberg, T. G. Maslova, A. B. Girshin, I. A. Popova, V. S. Saakov, O. F. Popova and G. A. Shirjayeva, Dokl. Akad. Nauk USSR, 154, 974 (1964).

35 D. I. Sapozhnikov, V. M. Kuturin, T. G. Maslova, M. V. Ulubekova, H. M. Nazarov, I. Yu. Artamkina and K. G. Semenuk, Dokl. Akad. Nauk USSR, 175, 1182 (1967).

36 D. I. Sapozhnikov and G. A. Kornjushenko, Plant Physiol. (USSR). 16, 1038 (1969).

37 D. I. Sapozhnikov, Z. M. Eidelman, N. V. Bazhanova, T. G. Maslova, O. F. Popova and G. A. Shirjayeva, Botan. Zh., 49, 1463 (1964).

${ }^{38}$ D. I. Sapozhnikov, T. G. Maslova, N. V. Bazhanova and O. F. Popova, Biophysics (USSR), 10, 349 (1965).

39 D. I. Sapozhnikov, L. V. Ivantsova, T. G. Maslova, I. A. Popova and O. Ya. Korolyova, Dokl. Akad. Nauk USSR, 189, 1135 (1969).

40 O. F. Popova and D. I. Sapozhnikov, Plant Physiol. (USSR), 19, 491 (1972).

41 O. F. Popova, Plant Physiol. (USSR), 18, 677 (1969).

42 O. F. Popova, Botan. Zh., 53, 978 (1968).

43 Z. M. Eidelman and A. S. Khodjayev, Dokl. Akad. Nauk USSR, 150, 928 (1963).

44 D. I. Sapozhnikov and O. Ya. Korolyova, Plant Physiol. (USSR), 18, 1273 (1971).

45 D. I. Sapozhnikov and N. V. Bazhanova, Dokl. Akad. Nauk USSR, 120, 1141 (1958).

46 D. I. Sapozhnikov, Z. M. Eidelman, D. Tolibekov and A. S. Khodjayev, Botan. Zh., 47, 1656 (1962).

${ }^{47}$ G. V. Krasichkova and D. I. Sapozhnikov, Dokl. Akad. Nauk Tadzhi. SSR, 11, 59 (1968).

48 A. Hager and H. Perz, Planta, 93, 314 (1970).

49 R. Willstätter and A. Stoll, Untersuchungen über die Assimilation der Kohlensäure, Springer, Berlin (1918).

50 H. Gaffron, Nature, 143, 205 (1939).

51 D. I. Sapozhnikov, Z. M. Fidelman, N. V. Bazhanova and O. F. Popova, Dokl. Akad. Nauk USSR, 127, 1128 (1959).

52 D. I. Sapozhnikov, The mechanisms of respiration, photosynthesis and nitrogen fixation, p. 299 Nauka (1967).

53 Z. M. Eidelman, D. I. Sapozhnikov, N. V. Bazhanova, O. F. Popova and G. A. Shirjayeva, Plant Physiol. (USSR), 7, 129 (1960).

${ }^{54}$ S. G. Petrenko, B. I. Bernstein, A. S. Okanenko, L. K. Ostrovskaya, T. A. Reingardt, I. P. Semenuk, I. V. Volkova and A. A. Jasnikov, Physiol. Biochem. Cult. Plants, 2,137 (1970).

55 Z. M. Eidelman, Usp. Sovrem. Biol., 53, 54 (1962).

56 D. I. Sapozhnikov, Usp. Sovrem. Biol., 64, 248 (1967).

57 T. W. Goodwin, The Comparative Biochemistry of the Carotenoids, p. 75, Chapman and Hall, London (1962).

58 Z. M. Eidelman, O. F. Popova, G. A. Shirjayeva and I. I. Chernjayeva, Eksperim. Botan., 16, 142 (1963).

59 J. R. Platt, Science, 129, 372 (1959).

${ }^{60} \mathrm{~K}$. Witt and C. Wolff, Z. Naturforch., 25b, 387 (1970).

61 J. Roquite, J. Phys. Chem., 70, 2699 (1966).

62 V. M. Kuturin, M. V. Ulubekova and H. M. Nazarov, Dokl. Akad. Nauk USSR, 186, 470 (1969).

63 F. A. Shneour and M. Calvin, Nature, 196, 339 (1962). 